\title{
Influência do tratamento térmico na redução do óxido de grafeno
}

\author{
Filho, J.C.S.; Soares, E.P.; Venancio, E. C.; Silva, S.C.; Takiishi, T \\ 1 Centro de Ciências e Tecnologia de Materiais, Instituto de Pesquisas Energéticas e Nucleares, São Paulo, Brasil. \\ 2 Centro de Engenharia, Modelagem e Ciências Sociais Aplicadas, Universidade Federal do ABC, Santo André, Brasil. \\ * e-mail: jorgecsilvaf@gmail.com / jorge.silva@ipen.br
}

\begin{abstract}
Resumo
A redução térmica de óxido de grafeno (GO) para o óxido de grafeno reduzido (rGO) é um passo importante no processamento para fabricar muitos materiais e dispositivos à base de grafeno. Neste trabalho, o GO foi sintetizado a partir do método de Hummers modificado, e as amostras foram tratadas termicamente à temperatura de $200^{\circ} \mathrm{C}$, com uma rampa de aquecimento de $10^{\circ} / \mathrm{min}$, e pressão de 10 $\mathrm{kPa}$, durante o tempo de 15 e 30 minutos. A caracterização de GO pelo DRX exibiu apenas um pico em $10^{\circ}$, do conjunto de planos GO (002), e a TGA demonstrou que o GO possui $10 \%$ de moléculas de água, voláteis e $26,77 \%$ de grupos com oxigênio. As amostras de rGO indicaram a perda de massa em 100 e $200^{\circ} \mathrm{C}$ na TGA e uma menor intensidade na absorção em $3489 \mathrm{~cm}^{-1}$ na FTIR.
\end{abstract}

\begin{abstract}
Thermal reduction of graphene oxide (GO) to reduced graphene oxide ( $\mathrm{rGO}$ ) is an important step in processing to manufacture many graphene based materials and devices. In this work, the GO was synthesized from the modified Hummer method, and samples were thermally treated at $473 \mathrm{k}$ at heating rate of $10 \% \mathrm{~min}$ and $10 \mathrm{kPa}$, during 15 and 30 minutes. The characterization of GO by XRD exhibited only a $10^{\circ}$ peak of the set of GO (002) planes, and the TGA shows that GO has $10 \%$ of water molecules, volatile and $26.77 \%$ of groups with oxygen. The rGO samples indicated mass loss at 100 and $200^{\circ} \mathrm{C}$ in TGA and a lower intensity of absorption at $3489 \mathrm{~cm} \mathrm{-1} \mathrm{in} \mathrm{FTIR}$
\end{abstract}

Keywords (Palavras chaves): óxido de grafeno reduzido, óxido de grafeno, tratamento térmico.

\section{Introdução}

Os nanocompósitos funcionais os quais são constituídos com grafeno, são de grande interesse para o campo científico [1]. A possibilidade de reduzir efetivamente óxido de grafeno (GO), mantendo ao mesmo tempo propriedades eletrônicas, é uma questão desafiadora para a ciência na qual necessita 0 entendimento para se ulitizar toda a versatilidade do grafeno para a produção de nanocompósitos em eletrônica e optoeletrônica [2]. Este sucesso em aplicações comerciais dos nanomateriais está relacionado com a eficácia em sinterizar, caracterizar e produzir materiais de elevada performance e qualidade em escala industrial [3].

É de conhecimento de que o grafeno apresenta excelentes propriedades eletrônicas, mecânicas e térmicas [4] e um dos métodos para a obtenção do grafeno é pela redução do óxido do grafeno (GO) [5] o qual pode ser realizado por reagentes químicos, radiação ionizante ou tratamento térmico. A reação de redução do GO para óxido de grafeno reduzido (rGO) é incompleta, e a partir disto, leva-se a formação do rGO, o qual possuí a vantagem de conter grupos com oxigênio, ou seja, epóxi, hidroxilas e carboxilas os quais fornecem hidrofilicidade do material, com isto, favorecem a dispersão em solventes polares, e a 
possibilidade de funcionalizá-los com diferentes e diversos materiais [6]. Neste trabalho foi realizado a síntese do GO através do método de Hummers modificado, e o tratamento térmico para redução GO em rGO foi realizado em atmosfera inerte, temperatura de $200^{\circ} \mathrm{C}$, pressão de $10 \mathrm{kPa}$, variando o tempo de tratamento. As amostras foram caracterizadas através da Análises Termogravimétricas (TGA), por Difração de raios-X (DRX), Espectroscopia no Infravermelho com transformada de Fourier (FTIR) e Microscopia Eletrônica de Varredura com Emissão de Campo (MEV-FEG).

\section{Metodologia}

\subsection{Preparação do GO}

O óxido de grafeno foi sintetizado a partir do pó do grafite (Merck Millipore com 99,99\% de pureza) utilizando o método de Hummers modificado [8]. O grafite foi adicionado em um balão contendo $\mathrm{H}_{2} \mathrm{SO}_{4} \mathrm{e}$ $\mathrm{NaNO}_{3}$. A mistura foi mantida sob agitação e, em seguida, adicionado, $18 \mathrm{~g} \mathrm{KMnO}_{4}$. Logo após, adicionase água destilada e a mistura é aquecida, sob agitação. Ao final da reação são adicionados $100 \mathrm{ml}$ de água deionizada e $\mathrm{H}_{2} \mathrm{O}_{2}$.

Posteriormente são realizadas: a lavagem, centrifugação, esfoliação e secagem da amostra.

\subsection{Preparação para o tratamento térmico de GO}

Foram separadas 2 amostras de óxido de grafeno em pó para o experimento na seguinte sequência: $\mathrm{rGO}_{1}=$

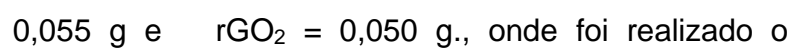
tratamento térmico com a temperatura de $200^{\circ} \mathrm{C}$, rampa de aquecimento de $10 \% \mathrm{~min}$, pressão de $10 \mathrm{kPa}$, respectivamente, e os $s$ tempos de 15 e 30 minutos para as amostras $\mathrm{rGO}_{1}$ e $\mathrm{rGO}_{2}$.

\section{Métodos de caracterização}

As Análises termogravimétricas (TGA), utilizou-se $3 \mathrm{mg}$ de cada amostra $\left(\mathrm{GO}, \mathrm{rGO}_{1}\right.$ e $\left.\mathrm{rGO}_{2}\right)$, e os materiais foram submetidos ao aquecimento a uma taxa de $20^{\circ} \mathrm{C} /$ min até atingir $800^{\circ} \mathrm{C}$.

\section{Resultados e discussões 4.1 Síntese de GO}

Análise Termogravimétrica (TGA) foi utilizada para medir a quantidade de perda de massa e porcentagem de perda de massa da amostra de GO, na Figura 1.

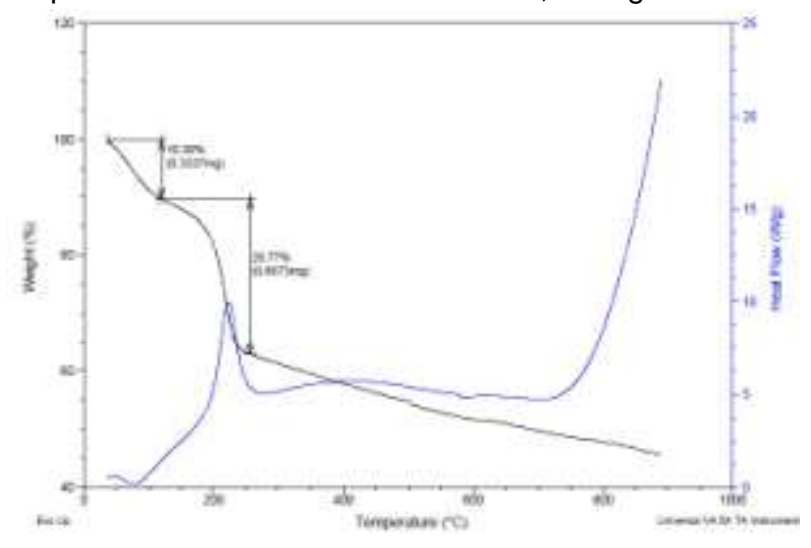

Figura 1: Análise de TGA do GO

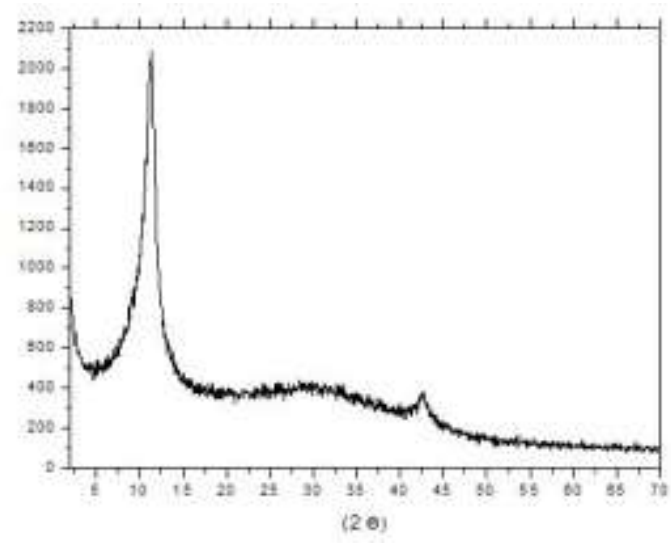

Figura 2: Difratograma de raios-X de GO

O difratograma de raios-X da amostra de GO (Figura 2) exibe apenas um pico em aproximadamente $10^{\circ}$ correspondente ao conjunto de planos GO (002) [8]. Este perfil de gráfico é esperado segundo a literatura [8,9], pois o GO nesta síntese contém 10,33\% de moléculas de água e voláteis e $26,77 \%$ de grupos com oxigênio, estas características demonstram que a síntese de do GO foi realizada com sucesso quando comparada com a literatura [9].

\subsection{Tratamento térmico rGO}

A Figuras 3 apresentam os resultados das análises termogravimétricas das amostras $\mathrm{rGO}_{1}$ e $\mathrm{rGO}_{2}$, comparando-as com amostra de GO. 


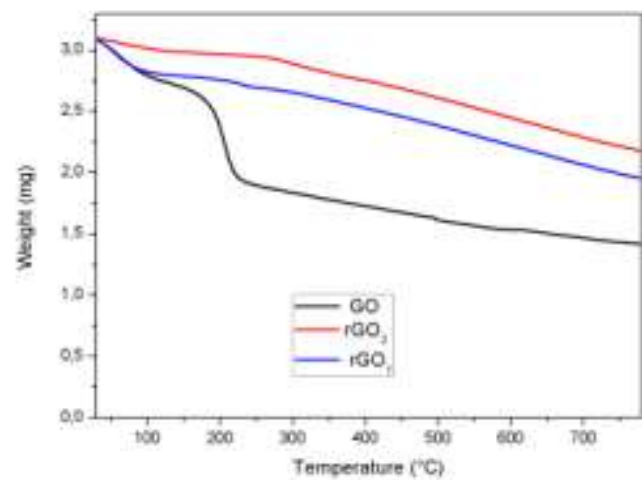

Figura 3: TGA das amostras: $\mathrm{GO}, \mathrm{rGO}_{1}$ e $\mathrm{rGO}_{2}$

As análises termogravimétricas das Figura 1 e Figura 3, demonstram que o GO é termicamente instável, e a partir do tratamento térmico, começa a perder massa à $100^{\circ} \mathrm{C}$ devido à evaporação de voláteis e moléculas de água adsorvida nas camadas da folha GO [10]. A maior perda de massa ocorre a $200^{\circ}$ devido à remoção de grupos funcionais contendo oxigênio tais como: $-\mathrm{OH}$, Epóxi e $\mathrm{COOH}$, ou seja, a perda de massa maior pode ser observado, juntamente com um sinal exotérmico da taxa de perda de massa (dw/dt, a Figura 1) a cerca de $200^{\circ} \mathrm{C}$, obtendo-se o $\mathrm{CO}$, o $\mathrm{CO}_{2}$ e vapor de água como subprodutos do processo de redução [11]. Com estes resultados, observamos que o tempo de tratamento térmico, ou seja, o tempo que amostra ficará exposta ao tratamento, influenciará significativamente nas propriedades do material, em vista que a quantidade de massa de água, voláteis e grupos com oxigênio, mudará dependendo do tratamento térmico, isto é observado pela mudança no perfil do gráfico da Figura 3, e também é confirmado na Espectroscopia do Infravermelho.

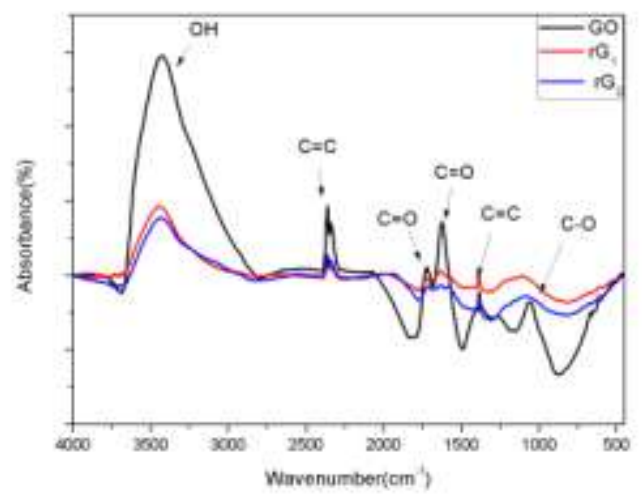

Figura 4: FTIR das amostras : $\mathrm{GO}, \mathrm{rGO}_{1}$ e $\mathrm{rGO}_{2}$
Pela análise de FTIR (Figura 4), o GO demonstra os picos de absorção em:3.681-3.489 $\mathrm{cm}^{-1}-\mathrm{OH}$ (hidroxila), $1.797 \mathrm{~cm}^{-1}-\mathrm{C}=\mathrm{O}$ (carbonila), $1.652 \mathrm{~cm}^{-1}$ $\mathrm{C}=\mathrm{C}$ (anel aromático), $1.300 \mathrm{~cm}^{-1} \mathrm{C}-\mathrm{O}$ (álcool), 1.130 $\mathrm{cm}^{-1}$ (epóxi) [12]. Após o tratamento térmico a intensidade da absorção a $3489 \mathrm{~cm}^{-1}$ (hidroxila) diminui para as amostras de rGO, ou seja, a intensidade relacionada a este pico característico da hidroxila reduz, comprovando a redução térmica do GO [13].

As Figuras 5(a) e 5(b) apresentam as micrografias obtidas utilizando Microscopia eletrônica de varredura (ampliação de 10000 vezes), tanto em relação ao GO e $\mathrm{rGO}_{1}$. O tamanho dos grãos de ambos é semelhante, embora a superfície do óxido de grafeno reduzido contenha menores imperfeições [14].

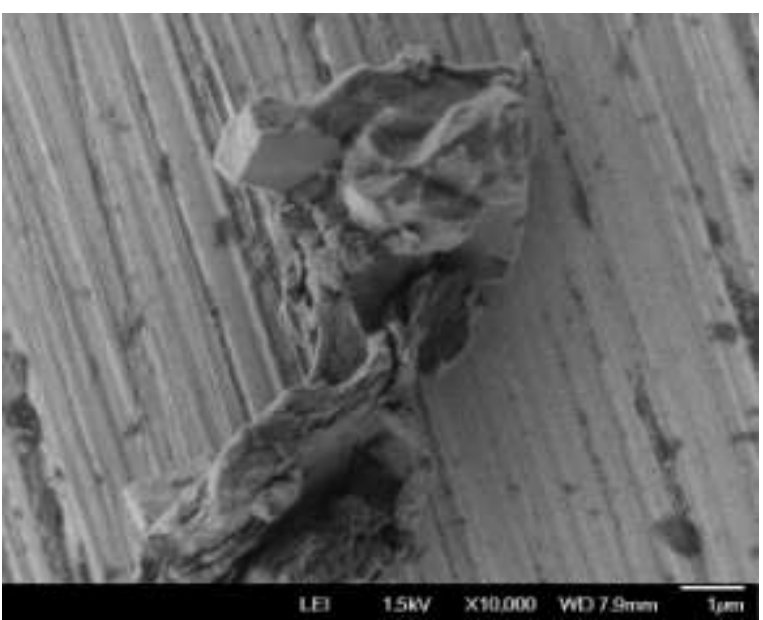

(a)

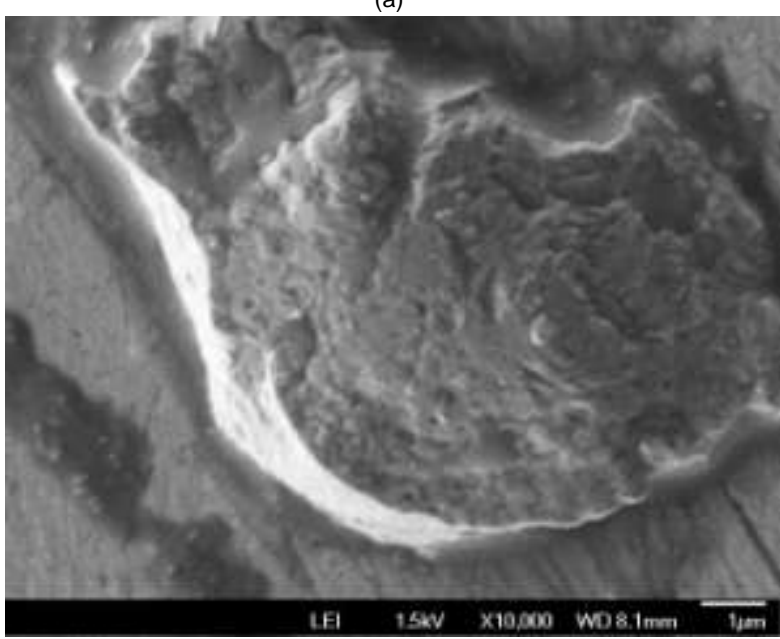

(b)

Figura 5: MEV-FEG (a) GO e (b) rGO

A micrografia do filme fino GO tem uma estrutura esponjosa, esta é uma indicação de que a grafite foi esfoliada durante o processo de oxidação. Isto é devido 
ao oxigênio e aos grupos de funcionais anexados ao óxido de grafite para formar GO [15].

\section{Conclusões}

A síntese de GO através do método de Hummer modificado foi confirmada através dos métodos de caracterizações (TGA, DRX e FTIR) e a redução térmica das amostras de GO em ambientes inertes sob aquecimento pode ocorrer em modo contínuo e controlado. Os resultados de TGA e FTIR, demonstram o processo de redução térmica do GO em rGO foi satisfatório, pois diminuiu-se os grupos funcionais contendo oxigênio no espectro FTIR de rGO e também confirmou a redução de GO para rGO.

\section{Agradecimentos}

Ao CNPQ (Processo Universal 800837/2014-2), o Centro de Ciência e Tecnologia de Materiais (CCTM), o Centro de Combustível Nuclear (CCN), a Mariana Araujo do laboratório de vidros e o IPEN-CNEN/SP para o suporte financeiro e a infra-estrutura disponibilizada para a realização deste projeto .

\section{.7. Referências}

[1] OLOWOJOBA, GB. ESLAVA, S. GUITIERREZ, ES. KINLOCH, AJ. MATTEVI, C. ROCHA, VG. TAULOR, AC. In situ thermally reduced graphene oxide/epoxy composites/ thermal and mechanical properties. Springer, v.6, p.1015-1022, 2016.

[2] LARCIPRETE, R. FABRIS, S. SUN, T. LACOVIG, P. BARALDI, A. LIZZIT, S. Dual Path Mechanism in the Thermal Reduction of Graphene Oxide. J. Am. Chem. Soc., v.133, p. 17315-17321, 2011.

[3] MARASCHIN, TG. Preparação de òxido de Grafeno e Óxido de Grafeno Reduzido e dispersão em matriz polimérica Biodegradável. Pontifícia Universidade Católica do Rio Grande do Sul. Porto Alegre, 2016.

[4] ZHANG, Y. MA, HL. ZHANG, Q. PENG, J. LI, J. ZHAI, M. YU, ZZ. Facile syntesis of well-dispersed graphene by y-ray induced reduction of graphene oxide. Journal of Materials Chemistry, v.22, p.13064-13069, 2012.
[5]. SERESHT, RJ. JAHANSCHAHI, M. RASHIDI, AM. GHORESYSHI, AA. Synthesis and Characterization of Thermally-Reduced Graphene. Iranica J. Energy \& Envirn. v.4, p.53-59, 2013.

[6]. YIN, K. LI, H. XIA, Y. BI., H. SUN, J. LIU, Z. SUN, L. Thermodynamic and Kinetic Analysis of Lowtemperature Thermal Reduction of Graphene Oxide. Nano-Micro Lett. v.3, p.51-55, 2011.

[8]. NAMVARI, M. BISWAS, CS. GALLUZZI, M. WANG, Q. DU, B. STADLER, FJ. Reduced graphene oxide composites with soluble copolymers having tailored lower critical solution temperatures and unique tube-like structure. Sci.Rep. v.7, p.44508, 2017.

[9]. HU, C. YU, T. LI, Y. Novel DOPO-Modified Graphene: Synthesis and Characterization. Journal of Nanoscience and Nanotechnology. v17, p.4894-4900, 2017.

[11]. GANGULY, A. SHARMA, S. PAPAKONSTANTINOU, P. HAMILTON, J. Proibing the Thermal Deoxygenation of Graphene Oxide using HighResoltuion In Situ X-ray-Based Spectroscopies. J.Phys. Chem. C. v.115(34), p.17009-17019, 2011.

[12]. FIM, FC. Síntese e propriedades de nanocompósitos de polietileno/nanolâminas de grafeno obtidos através de Polimerização In Sisu. Tese de Doutorado. Programa de Pós-Graduação em Ciencias dos Materiais, Universidade Federal do Rio Grande do Sul, Porto Alegre, 2012.

[13]. OSSONON, BD. BÉLANGER, D. Synthesis and characterization of sulfophenyl-functionaized reduced graphene oxide sheets. RSC ADV. v.7, p.27224, 2017. [15]. DREWNIAK, S. MUZYKA, R. STOLARCZYK, A. PUSTELNY, T. MORANSKA, MK. SETKIEWICZ, M. Studies of Reduced Graphene Oxide and Graphite Oxide in the Aspect of Their Possible Application in Gas Sensors. Sensors. v.16, p.103, 2016.

[16] LIU, F. CAO, Y. Yi, M. XIE, L. HUANG, W. TANG, N. ZHONG, W. DU, Y. Thermostability, Photoluminescence, and Electrical Properties of Reduced Graphene Oxide-Carbon Nanotube Hybrid Materials. Crystals. v. 3, p.28-37, 2013. 